\title{
'|||||||||||||||||||||||||||||||||||||||||||||||||||||||||||||||||||||.
}

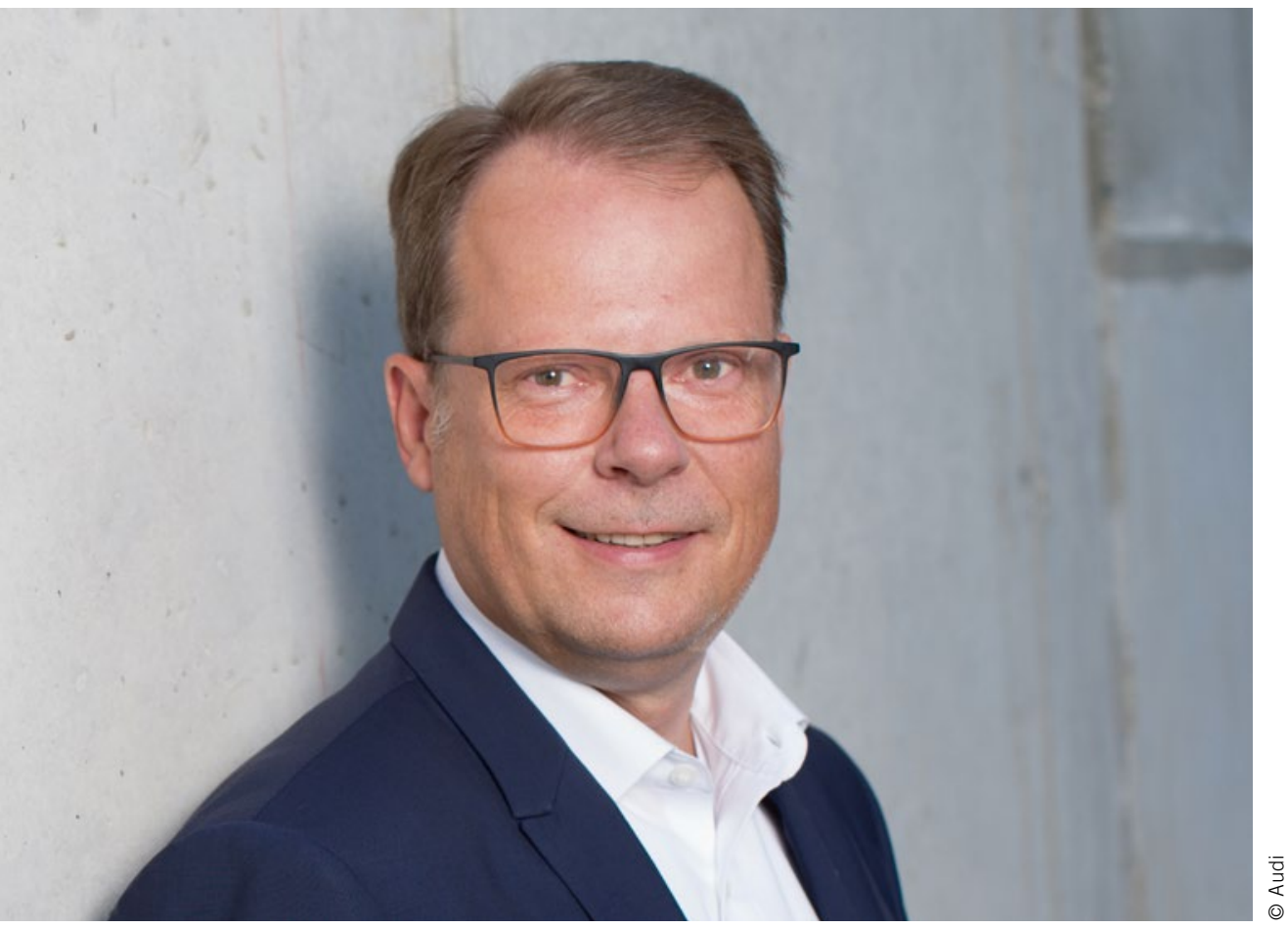

Dr.-Ing. Peter Mertens Member of the Board of Management of Audi AG, Technical Development, ATZ Advisory Board

\section{Automotive Autonomy}

Technological change is the driving force behind the automotive industry. It has been securing the industry's existence since 1886. At Audi, affinity for technology and capacity for transformation are summed up in its motto "Vorsprung durch Technik", or progress through technology. quattro and TFSI, laser light and lightweight design are all examples of Vorsprung durch Technik. They represent major technological development steps. Audi AI is now going one step further. It marks the beginning of the age of artificial intelligence (AI) in vehicles. "Audi Artificial Intelligence” describes intelligent, self-learning assistance systems and autonomous driving. It stands for the connectivity of a car with its environment - no matter whether this is another road user, a traffic control system, a parking garage, or a charging station. This at last will make automated driving possible, which itself will revolutionize mobility.

Autonomous vehicles require artificial intelligence as one of the key technologies. Almost in real time, AI will match data from the infrastructure with data from other road users. Audi AI will enable the fully connected car to look much farther ahead than the systems previously used - a time of around $10 \mathrm{~s}$ is already possible today. Or to put it another way: the car of the future will for the first time be able to make predictions. And it is this that will make level 3 automated driving possible, in the future forming the basis for level 4 and level 5, fully automated and, as the final step, autonomous driving.
For drivers, autonomous mobility means additional comfort, freedom, and safety. Freedom in the sense that autonomous driving will enable drivers of a fully connected car to use their time more freely. They can gradually take their hands off the steering wheel and instead of driving they can work, communicate, or just relax. What is more, they will have more time for themselves, because an autonomous car will relieve them of many mundane, routine activities, like parking or driving through a car wash.

As for safety: more than $90 \%$ of all road accidents today are caused by human error. For that reason, automated driving promises a significant reduction in the number of road traffic accidents, a view that is also supported by politicians. For example, the German Federal Government's "Ethics Commission on Automated and Connected Driving” wrote in its final report in June 2017: “As the 'perception' of the vehicle's surroundings becomes increasingly perfected, there is likely to be an ever better differentiation of road users, obstacles, and hazardous situations. This makes it likely that it will be possible to significantly enhance road safety." That is also Audi's expectation with its many different assistance systems.

Digitization and connected vehicles make mobility safer and more comfortable. Automated driving is the next milestone in the history of the automobile that spans more than 130 years. 